\title{
Enjoying to Work
}

This special issue is dedicated to a well-known theoretical computer scientist, Professor Mario de Jesús Pérez-Jiménez, the head of the Research Group on Natural Computing at Seville University, on the occasion of his 65 th birthday.

Mario is a unique combination of enthusiasm and mathematical talent, scientific devotion and altruism. He contributed massively and in a significant way to research in computer science. During the last decade his research was focused on membrane computing, where he contributed fundamental results especially related to computational complexity as well as results related to many other theoretical research questions, to applications in biology and eco-systems, and to programming. He created around him an impressive research group in natural computing specializing in membrane computing. Since 2004, his group organizes every year the Brainstorming Week on Membrane Computing - in this way Seville became the place of "pilgrimage" for researchers in membrane computing coming there from all over the world.

As a natural recognition for his contributions to computer science, Mario was elected a member of the Informatics Section of Academia Europaea.

Mario loves his work - as a matter of fact he often explicitly reminds his group to "enjoy what we do". His enthusiasm is contagious, it truly energizes people around him - it is a real pleasure and excitement to collaborate with Mario.

Happy Birthday and thank you dear Mario!

Following Mario's scientific passion, the main theme of this special issue is membrane computing. More specifically, this special issue contains a selection of papers produced during the 12th Brainstorming Week on Membrane Computing, held in Seville, from February 3 to February 7, 2014, augmented by six invited papers written by authors who are close to Mario and his Seville group. All papers went through a thorough refereeing procedure. Together they provide a representative sample of current research in membrane computing which covers a broad spectrum of directions ranging from theoretical to applied.

The Brainstorming Weeks on Membrane Computing are well documented in the literature - the interested reader can find details at the membrane computing website, at http://ppage.psystems . eu. 
We thank the authors for contributing papers to this special issue and the referees for their help in the selection process. We are also indebted to Prof. D. Niwinski for his encouragement to publish this special issue in Fundamenta Informaticae.

Marian Gheorghe, Sheffield, UK Gheorghe Păun, Bucharest, Romania Agustín Riscos-Núñez, Seville, Spain Grzegorz Rozenberg, Leiden, The Netherlands

Editors 